\title{
Retention of cue-based associations in the water maze is time-dependent and sensitive to disruption by rotating the starting position
}

\author{
Anne-Marie T. McGauran, Deirdre Harvey, Lorretto Cunningham, \\ Sarah Craig, Sean Commins* \\ Department of Psychology, National University of Ireland, Maynooth, Co. Kildare, Ireland
}

Received 13 June 2003; received in revised form 1 September 2003; accepted 1 September 2003

\begin{abstract}
Research has focused mainly on the acquisition phase of spatial tasks, while retention has been relatively ignored. In three experiments, we determine the type of information that is retained in spatial memory using the water maze task. In experiment 1 , we demonstrate that by rotating the distal cues $180^{\circ}$ post-acquisition Wistar rats search in the opposite area to where the platform should be. This search continues for a maximum of $30 \mathrm{~s}$. We then demonstrate (experiment 2$)$ that by rotating the starting position $\left(180^{\circ}\right.$ post-acquisition) animals remain at the starting-point for $10 \mathrm{~s}$. They then commence searching in the platform area. In experiment 3, we demonstrate that rotations of distal cues and starting position post-acquisition impair retention of the platform's location. We suggest that the association between the configuration of distal cues and platform location is retained in memory but the association is fragile and sensitive to disruption.
\end{abstract}

(C) 2003 Elsevier B.V. All rights reserved.

Keywords: Water maze; Retention; Cue-rotation; Starting position rotation; Spatial memory

\section{Introduction}

Learning and memory, as measured by changes in an animal's behaviour is composed of a number of different processes which overlap each other, these processes include acquisition, consolidation and retrieval [1]. Spatial learning and memory can also be considered in terms of acquisition, consolidation and retrieval. One tool described 20 years ago to investigate spatial learning and memory is the water maze [2,3]. Much of the research has focused on acquisition phase of spatial tasks such as the water maze [4]. Further research has examined disruption of acquisition via pharmacological [5] behavioural [6] or genetic manipulation [7] to probe the brain structures and mechanisms which underlie spatial learning.

Recent studies using the water maze have shown that animals use multiple learning strategies to acquire the water maze task $[8,9]$. These strategies can be based on the relationship between distal cues and the location of the platform (allocentric) or can be based on the relationship between the animal and the platform (egocentric). These two strategies

\footnotetext{
* Corresponding author. Tel.: +353-1-7086182; fax: +353-1-7084767.

E-mail address: Sean.Commins@may.ie (S. Commins).
}

also interact with each other [10] and may involve different brain areas (e.g. [11]).

In contrast to acquisition, consolidation or retrieval of long-term spatial memory has received little attention despite the importance of these processes in models of hippocampal functioning $[12,13]$. This may be due to lack of sensitive behavioural methodologies. Currently, consolidation and retrieval of spatial memories in the water maze task are examined by a probe test (for example, see [14,15]). This typically consists of a single $60 \mathrm{~s}$ probe trial, where the platform has been removed. The percentage time spent in the target quadrant (where the platform should be) determines retention performance [14].

If multiple forms of spatial information are used to acquire the task then by extension multiple forms of spatial information may be retained to form long-term memories. A few studies have demonstrated that animals have the ability to retain a learned task over a long period of time. Bolhuis et al. [16] and Mumby et al. [17], for example, demonstrate that in the water maze task a rat's memory for the location of the platform is retained for at least 14 weeks. While Van Groen et al. [18] demonstrate retention of the task following 12 months. What type of information is retained? Abel and Lattal [1] suggest that retrieval may share similar mechanisms with acquisition. If an association between the distal 
cues and the platform location is built up during acquisition [19], is this cue-dependent information stored and subsequently used during retrieval? Alternatively are other strategies retained? Animals may use strategies that rely more on retained procedural information such as dead-reckoning or path integration ([20], see also [21]). The experiments presented in this paper attempt to investigate searching strategies employed during retention and aim to disassociate the types of information that are stored in spatial memory. If cue-dependent information is stored then we would predict that rotation of cues 7 days post-acquisition should lead to rotation in searching strategy in the retention probe task. However, if what is stored is simply a set of learned movements (procedural information) then rotation of the starting position of the animals by $180^{\circ} 7$ days post-acquisition would lead to an initial search in the opposite quadrant $\left(180^{\circ}\right.$ from where the platform should be). We further predict that upon failure to locate the platform here animals would use cue-information and return to search in the platform area.

If a conflict arises between the two types of strategies we predict that cue-information would be dominant. Therefore, rotation of both the cues (by $180^{\circ}$ ) and starting position would lead to a dominant cue-based retention search strategy (searching in opposite area to where the platform should be in the probe task). Although the 60 -s probe trial is the standard tool to assess retention, we will break this $60 \mathrm{~s}$ into 10 -s time bins to examine the subtle changes in searching strategies.

\section{Materials and methods}

\subsection{Animals}

Male Wistar rats (200-300 g; Bioresources Unit, University of Dublin, Trinity College) aged approximately 3 months were used as subjects. Rats were housed three per cage and were kept in a temperature-controlled room which was maintained on a fixed light-dark cycle (07:00-19:00 h). All rats were given free access to food and drink. All rats were well handled before experimentation and testing was carried out during the light phase.

\subsection{Apparatus and procedure}

The water maze was a black circular pool $(1.7 \mathrm{~m}$ diameter; $35 \mathrm{~cm}$ deep; water $20 \pm 1{ }^{\circ} \mathrm{C}$ ) filled to $31 \mathrm{~cm}$. Rats can escape the water by climbing on to a hidden platform $(29 \mathrm{~cm} \times 9 \mathrm{~cm})$. The hidden platform was placed in the North Eastern quadrant of the pool and submerged $2 \mathrm{~cm}$ below water surface so it was invisible at water level; the location of the platform was fixed during the acquisition of all experiments. A curtain surrounded the water maze at a distance of $50 \mathrm{~cm}$ from the pool wall. Distal cues included two $(60 \mathrm{~W})$ lights suspended from the ceiling. One light was suspended from the NE corner, and the other from the NW position.
Both lights were at a distance of $75 \mathrm{~cm}$ from the pool wall and at angles of approximately $60^{\circ}$. A rectangular sheet of black paper $(55 \mathrm{~cm} \times 81 \mathrm{~cm})$ stuck to the curtain on the eastern side of the pool was also used as a cue. A computerised digital tracking system (EthoVision) recorded escape latencies and distance travelled during each trial (Noldus Information Technology, Wageningen, The Netherlands).

\subsubsection{Experiment 1}

Rats $(n=15)$ received four trials per day for 4 days to acquire the task. Rats were allowed $60 \mathrm{~s}$ to find the platform; otherwise the rat was led to the platform by the researcher. The rat remained on the platform for $20 \mathrm{~s}$. The inter-trial interval was $10 \mathrm{~s}$. All rats entered the maze from a pseudorandom starting position (North, South, East or West).

Retention was assessed 7 days post-acquisition. Rats were then randomly assigned to one of two groups; controls ( $n=$ 7) or cue-rotated group $(n=8)$. Retention for both groups was assessed by removing the platform and allowed to swim for $60 \mathrm{~s}$. For control group each rat was placed into the water maze at NW position. The distal-cue layout remained the same as during the acquisition phase. For cue-rotated group, each rat was again placed into the water at the NW position but the distal cues were rotated by $180^{\circ}$, so that one light was now suspended from the SW corner, and the other from the SE position. The sheet of black paper was now positioned on the western side.

Various measures of retention were used. Percentage time spent in the platform area (on total time spent in the pool, i.e. $60 \mathrm{~s}$ ) was used to measure retention. This was defined by a circular area centred at the platform with a radius of approximately $27 \mathrm{~cm}$ (body length of a rat). Percentage time spent (again on total time spent in the pool) in three other equivalent locations (NW, SW and SE) was also calculated.

Percentage time spent (again on the total time spent in the pool) at the edge of the pool, in an area termed the 'Panic Corridor' was also measured. This was defined by a circular area, approximately $20 \mathrm{~cm}$ in width, inside the pool. Confused or nervous rats have a tendency to swim near the wall of the pool [22]. This response is defined as thigmotaxis by [23] who described it as a species-typical fear behaviour [24]. It is also thought to result from an impairment of learning certain aspects of spatial navigation [25].

\subsubsection{Experiment 2}

Rats $(n=15)$ received a similar training schedule to experiment 1. However, all rats entered the maze from fixed-starting position (NW).

Retention was again assessed 7 days post-acquisition. These rats were randomly assigned to one of two groups; controls $(n=7)$ or start-rotated group $(n=8)$. Retention for both groups was assessed by removing the platform and allowed to swim for $60 \mathrm{~s}$. The distal cue layout remained the same as during the acquisition phase. For the control group each rat was placed in the water from the NW posi- 
tion (as during acquisition). For start-rotated group, each rat was placed into the water at the SE position.

The various retention measures used in experiment 1 were also used here.

\subsubsection{Experiment 3}

Rats $(n=15)$ received a similar training schedule to experiment 1. However, all rats entered the maze from fixed-starting position (SW). Retention was again assessed 7 days post-acquisition. These rats were randomly assigned to one of two groups; controls $(n=7)$ or start- and cue-rotated group $(n=8)$. Retention for both groups was assessed by removing the platform and allowed to swim for $60 \mathrm{~s}$. For the control group, each rat was placed into the maze at SW. The external-cue layout remained untouched by the experimenters. For start- and cue-rotated group, each rat was placed into the SE quadrant (rotating the starting point by $90^{\circ}$ ) and the distal cues were rotated by $180^{\circ}$.
Again the various retention measures used in experiment 1 were also used here.

\subsection{Statistics}

A series of repeated ANOVAs were used. Where appropriate independent $t$-tests were also used. All statistics were carried out using SPSS (version 10).

\section{Results}

\subsection{Experiment 1: effects of cue-rotation on retention}

All rats acquired the water maze task. The mean escape latencies decreased over the 4 days from $46.77 \pm 2.67 \mathrm{~s}$ on day 1 to $22.29 \pm 2.24 \mathrm{~s}$ on day 4 . A one-way ANOVA confirmed that an overall significance was found between the

(a)
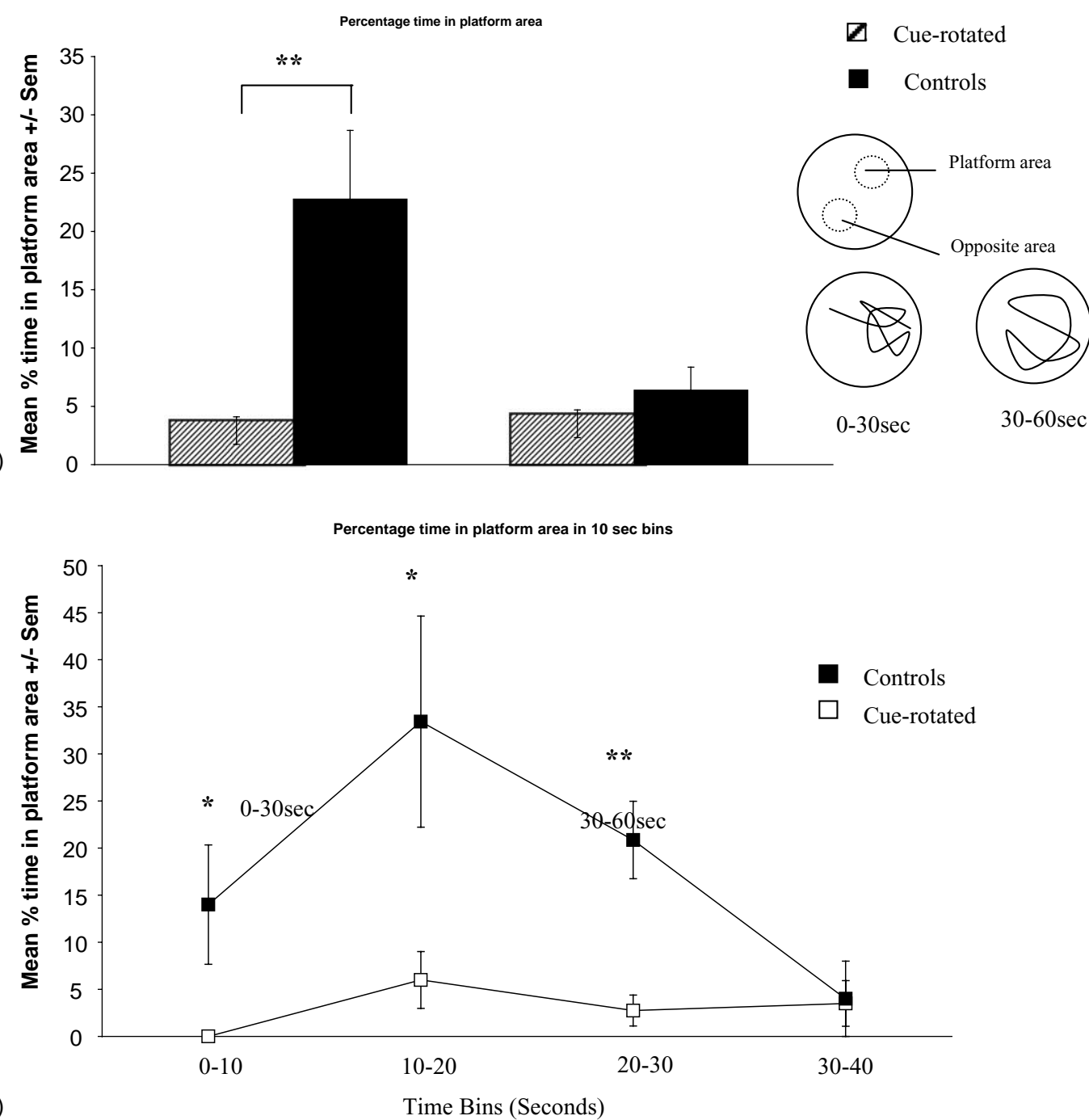

Fig. 1. (a) Bar chart demonstrating the percentage time spent by control and cue-rotated groups in the platform area (see inset) for the first and last $30 \mathrm{~s}$ of the retention phase. (b) A line chart displaying the percentage time spent by control and cue-rotated groups in the platform area for the first $40 \mathrm{~s}$ of retention trial (in 10-s bins). Representative swim paths for the controls for the first and last $30 \mathrm{~s}$ are displayed in (a). 
4 days $(F=16.798$, d.f. $=3,240, P<0.001)$. Subsequent post hoc tests (Tukey, $P<0.05$ ) demonstrated that the mean escape latencies on days 3 and 4 were significantly shorter than on day 1.

Retention of the water maze was assessed 7 days postacquisition. Rats were randomly assigned to one of two groups: controls $(n=7)$ or cue-rotated group $(n=8)$. Fig. 1a reveals that the control group spent significantly more time swimming in the platform area (NE, 22.76 $\pm 5.8 \%$ ) than the cue-rotated group $(2.91 \pm 1.1 \%)$ in the first $30 \mathrm{~s}$ $(t=-3.536$, d.f. $=13, P<0.01)$. However, there was no significant difference $(t=-1.280$, d.f. $=13, P>$ $0.05)$ in the amount of time spent searching in the platform area by either the cue-rotated or control group $(3.5 \pm 1.2 \%$ and $6.3 \pm 1.9 \%$, respectively) between 30 and $60 \mathrm{~s}$. We further analysed the first $30 \mathrm{~s}$ in $10-\mathrm{s}$ bins. We found that the controls spent significantly more time searching in the platform area in the first $10 \mathrm{~s}(t=-2.374$, d.f. $=13$, $P<0.05), 10-20 \mathrm{~s}(t=-2.511$, d.f. $=13, P<0.05)$ and 20-30s $(t=-4.296$, d.f. $=13, P<0.01)$ when compared to the cue-rotated group (see Fig. 1b). Rotation of the cues $180^{\circ}$ clearly impaired the search strategy of this group. Where did the cue-rotated group search for the platform?

Fig. 2a shows that the cue-rotated group spent significantly more time searching for the platform in the opposite area (SW, $11.58 \pm 2.73 \%$ ) than the control group $(2.57 \pm 0.9 \%)$ in the first $30 \mathrm{~s}(t=2.951$, d.f. $=13, P<$ $0.01)$. Again this effect was lost after $30 \mathrm{~s}(t=-0.483$, d.f. $=13, P>0.05$ ). We further analysed the first $30 \mathrm{~s}$ in 10 -s bins and found that the cue-rotated group spent significantly more time searching in the opposite area between 20 and $30 \mathrm{~s}(t=2.651$, d.f. $=13, P<0.05)$ when compared to the controls (see Fig. 2b). (a)

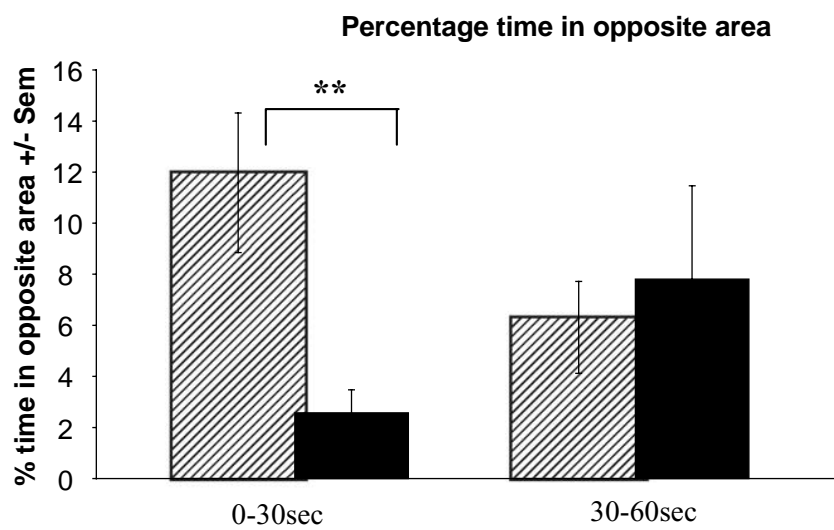

D Cue-rotated
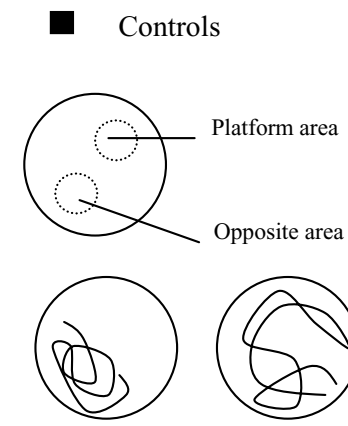

$0-30 \mathrm{sec}$

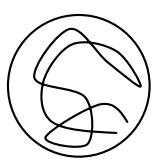

$30-60 \mathrm{sec}$

Percentage time in opposite area in 10 sec bins

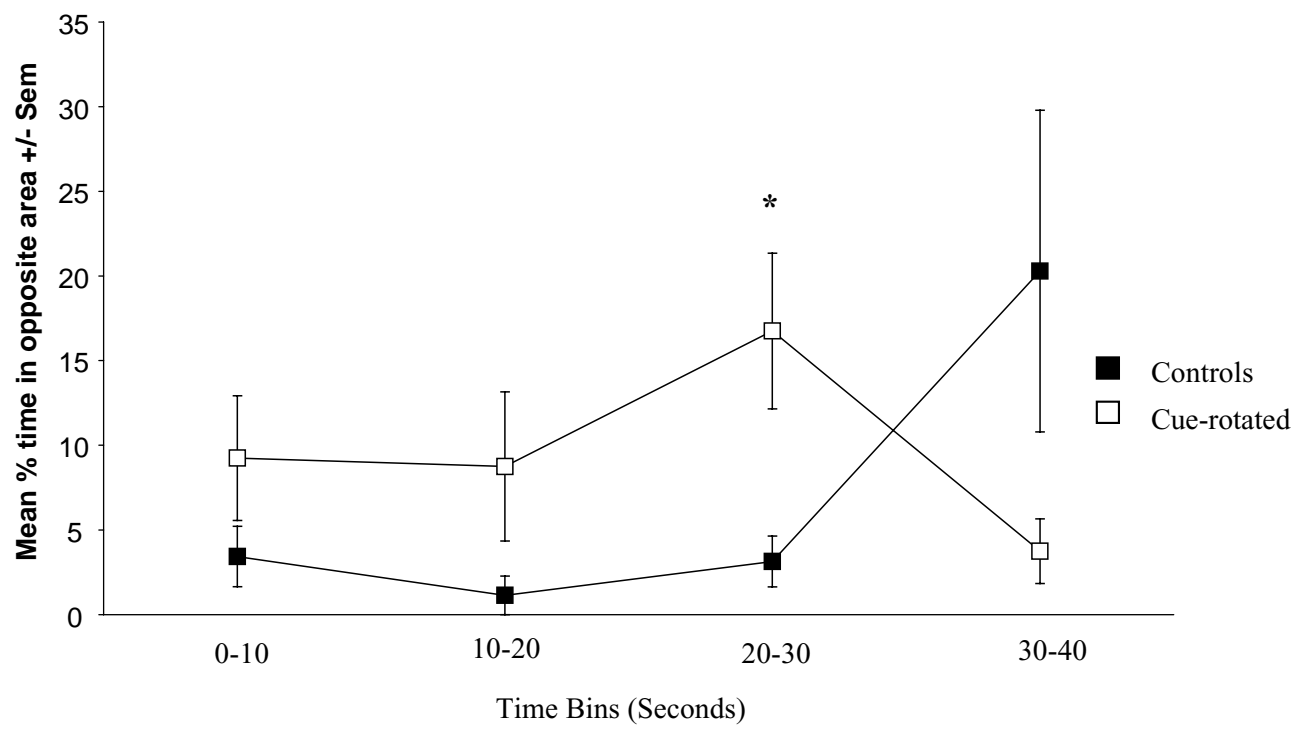

Fig. 2. (a) A bar chart displaying the percentage time spent by control and cue-rotated groups in the opposite area (see inset) for the first and last $30 \mathrm{~s}$ of the retention phase. (b) A line graph demonstrating the percentage time spent in the opposite area by the control and cue-rotated groups for the first $40 \mathrm{~s}$ of retention trial (in 10-s bins). Representative swim paths for the cue-rotated group for the first and last $30 \mathrm{~s}$ are displayed in (a). 
Percentage time in platform area

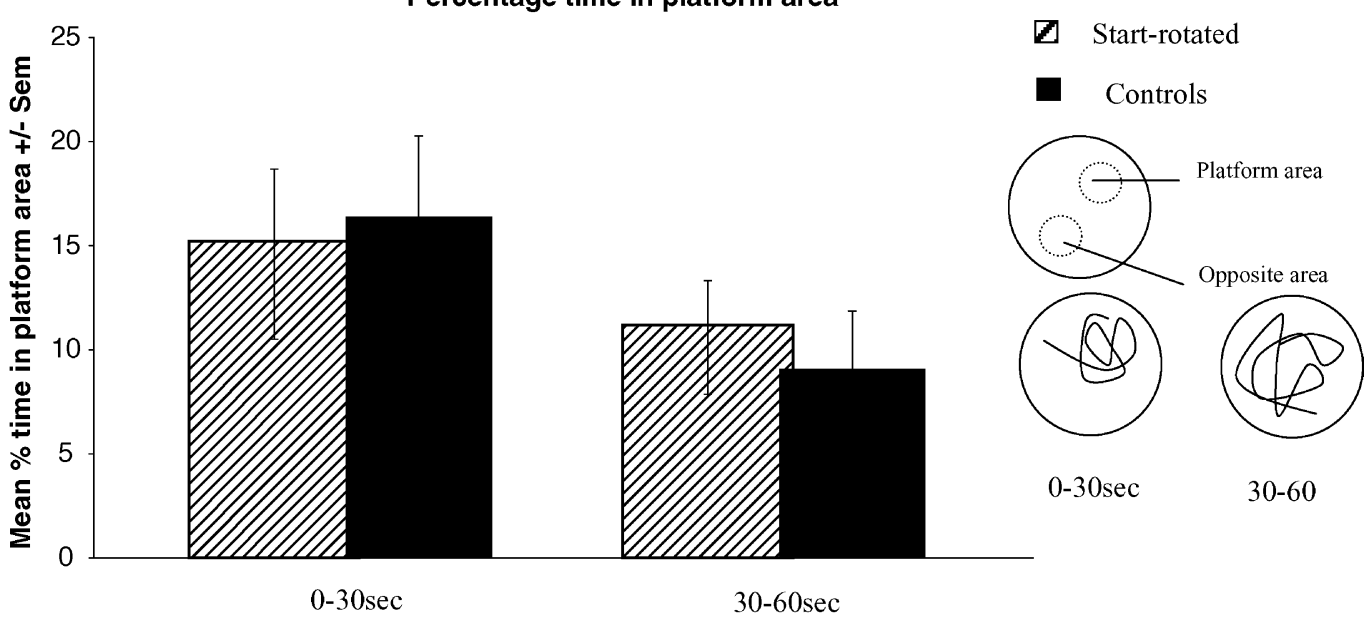

Percentage time in platform area in time bins

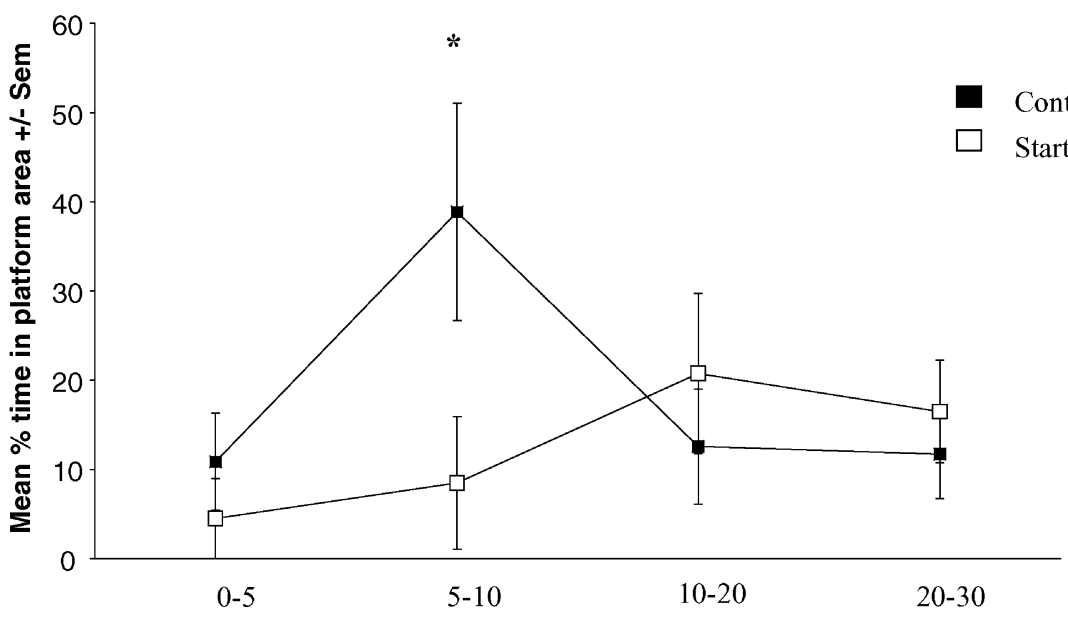

(b)

Time Bins (Seconds)

Fig. 3. (a) Bar chart demonstrating the percentage time spent by control and start-rotated groups in the platform area (see inset) for the first and last $30 \mathrm{~s}$ of the retention phase. (b) A line chart displaying the percentage time spent by control and start-rotated groups in the platform area for the first $30 \mathrm{~s}$ of retention trial (in 5- and 10-s bins). Representative swim paths for the controls for the first and last $30 \mathrm{~s}$ are displayed in (a).

\subsection{Experiment 2: effects of start-rotation on retention}

In experiment 2, all rats acquired the water maze task. The mean escape latencies decreased over the 4 days from $46.98 \pm 2.5 \mathrm{~s}$ on day 1 to $14.21 \pm 1.37 \mathrm{~s}$ on day 4 . A one-way ANOVA confirmed that an overall significance was found between the 4 days ( $F=33.622$, d.f. $=3,240, P<0.001)$. Subsequent post hoc tests (Tukey, $P<0.05$ ) demonstrated that the mean escape latencies on days 2,3 and 4 were significantly shorter than on day 1 .

Retention of the water maze was assessed 7 days post-acquisition. Rats were randomly assigned to one of two groups: controls $(n=7)$ or start-rotated group $(n=8)$. Fig. 3a reveals that there was no significant difference in the time spent swimming in the platform area by the start-rotated $(14.5 \pm 4.0 \%)$ compared to the control group $(16.38 \pm 3.88 \%)$ in the first $30 \mathrm{~s}(t=0.316$, d.f. $=13$,
$P>0.05)$. Again no significant differences $(t=0.393$, d.f. $=13, P>0.05)$ were found between either group in the $30-60 \mathrm{~s}$ period. However, we further analysed the initial 30-s period of retention (broken into 5- and 10-s bins). Fig. 3 b demonstrates that in the 5-10-s period, the controls spent significantly more time $(38.8 \pm 12.1 \%)$ swimming in the platform area compared to the start-rotated group $(8.5 \pm 7.4 \%, t=-2.191$, d.f. $=13, P<0.05)$. Rotating the starting position of the animals had a small but significant effect on their search strategy when the animals were initially place into the pool. Where did the start-rotated group search during this initial 10 -s period?

Fig. 4a demonstrates that the start-rotated group did not search significantly more in the opposite area (SW) when compared to controls in the first $30 \mathrm{~s}$ of retention $(t=-1.702$, d.f. $=13, P>0.05)$. When this time period was broken into 5- and 10-s bins (Fig. 4b) again no differ- 
(a)

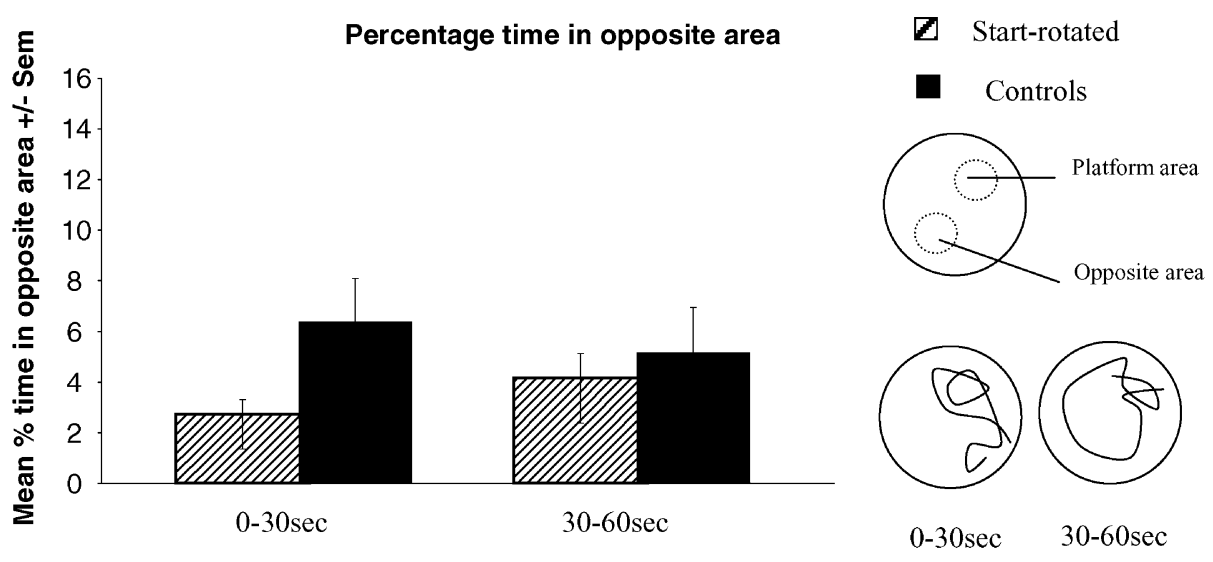

Percentage time in opposite area in time bins

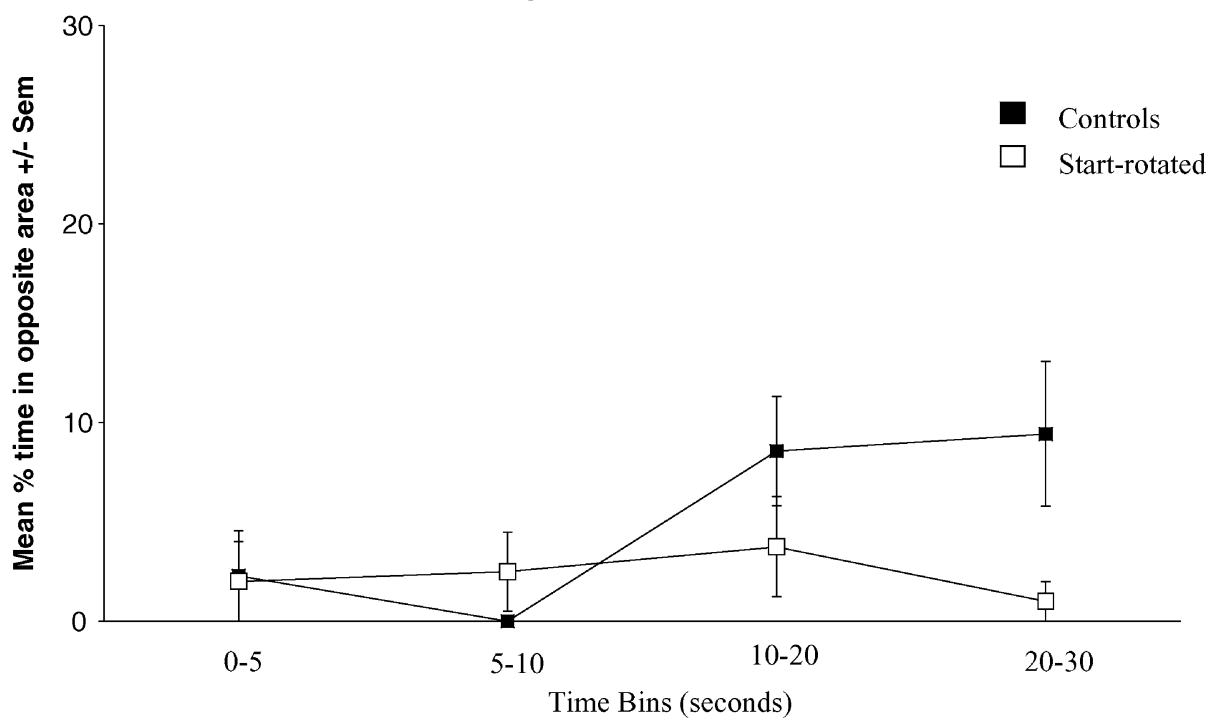

Fig. 4. (a) A bar chart displaying the percentage time spent by control and start-rotated groups in the opposite area (see inset) for the first and last $30 \mathrm{~s}$ of the retention phase. (b) A line graph demonstrating the percentage time spent in the opposite area by the control and cue-rotated groups for the first $30 \mathrm{~s}$ of retention trial (in 5- and 10-s bins). Representative swim paths for the cue-rotated group for the first and last $30 \mathrm{~s}$ are displayed in (a).

ences were observed between the groups. We then defined a 'panic corridor' at the side of the pool to eliminate the possibility that during the initial 10 -s period the start-rotated group swam around the side of the pool (see Fig. 5a inset). Fig. 5a reveals that there was no significant difference in the percentage time spent in the 'panic corridor' between the start-rotated group when compared to the controls $(t=1.537$, d.f. $=13, P>0.05)$. We then divided the 'panic corridor' into four areas (see Fig. 5b inset) to examine the percentage time spent where the animals were initially placed into the pool. We found (see Fig. 5b) that in the first $10 \mathrm{~s}$, the start-rotated group spent significantly more time at their starting position $(47.75 \pm 8.9 \%)$ than the controls spent at their respective starting position $(16 \pm 7.7 \%$, $t=-2.645$, d.f. $=13, P<0.05)$. A one-way ANOVA confirmed that there was an overall significant difference in the mean percentage time spent by the start-rotated group in the four equivalent areas (see Fig. 5c inset, $F=11.559$, d.f. $=3,31, P<0.01$ ). Subsequent post hoc tests (Tukey) revealed that the start-rotated group spent significantly more time in their starting position (SE, see Fig. 5c) when compared to the other three equivalent areas. A similar analysis was conducted for the controls (see Fig. 5d). This revealed that the controls spent equivalent amount of time in all four areas in the initial $10 \mathrm{~s}(F=1.520$, d.f. $=3,27$, $P>0.05)$.

\subsection{Experiment 3: effects of cue-rotation and start-rotation on retention}

In experiment 3 , all rats $(n=15)$ acquired the water maze task. The mean escape latencies decreased over the four days from $54.23 \pm 1.7 \mathrm{~s}$ on day 1 to $16.59 \pm 1.8 \mathrm{~s}$ on day 4 . A one-way ANOVA confirmed that an overall significance was found between the 4 days $(F=45.49$, d.f. $=3,240$, $P<0.001$ ). Subsequent post hoc tests (Tukey, $P<0.05$ ) demonstrated that the mean escape latencies on days 2, 3 and 4 were significantly shorter than on day 1 . 

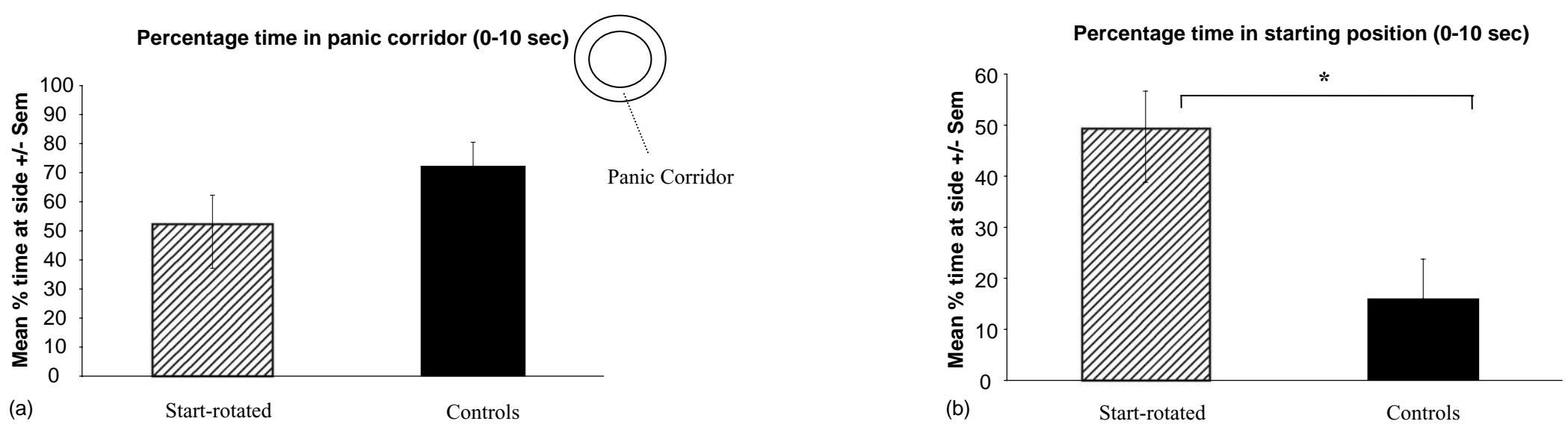

Control (NW)

Percentage time spent inpanic corridor (Start-rotated, 0-10 sec)
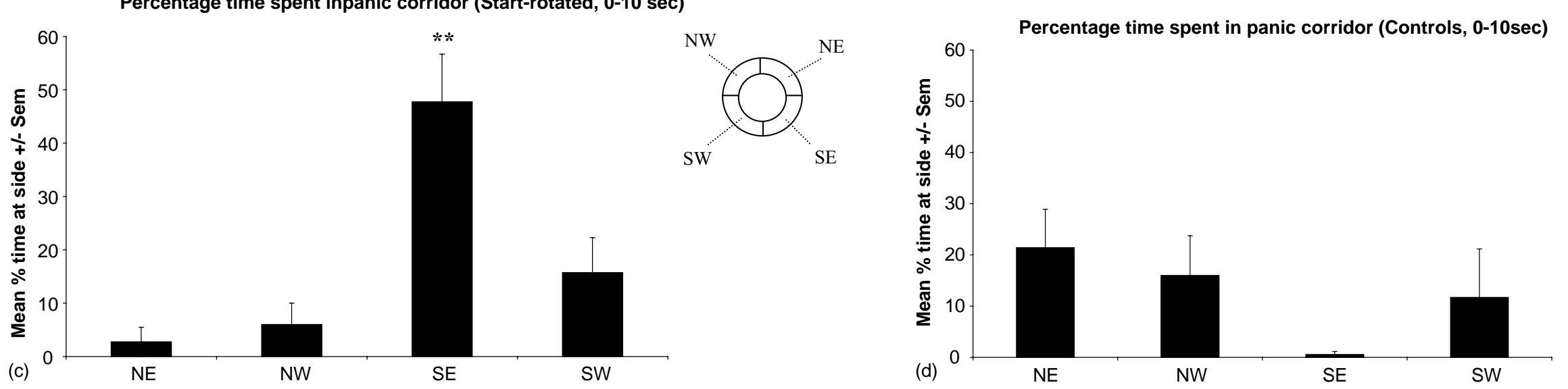

Fig. 5. (a and b) Bar charts displaying the percentage time spent swimming by the control and start-rotated groups in the panic corridor (a) and where they were placed into pool (b) for the first $10 \mathrm{~s}$. (c and d) Bar charts demonstrating the percentage time spent swimming in the four areas (see inset) of the panic corridor by the start-rotated group (c) and controls (d) for the first $10 \mathrm{~s}$. 


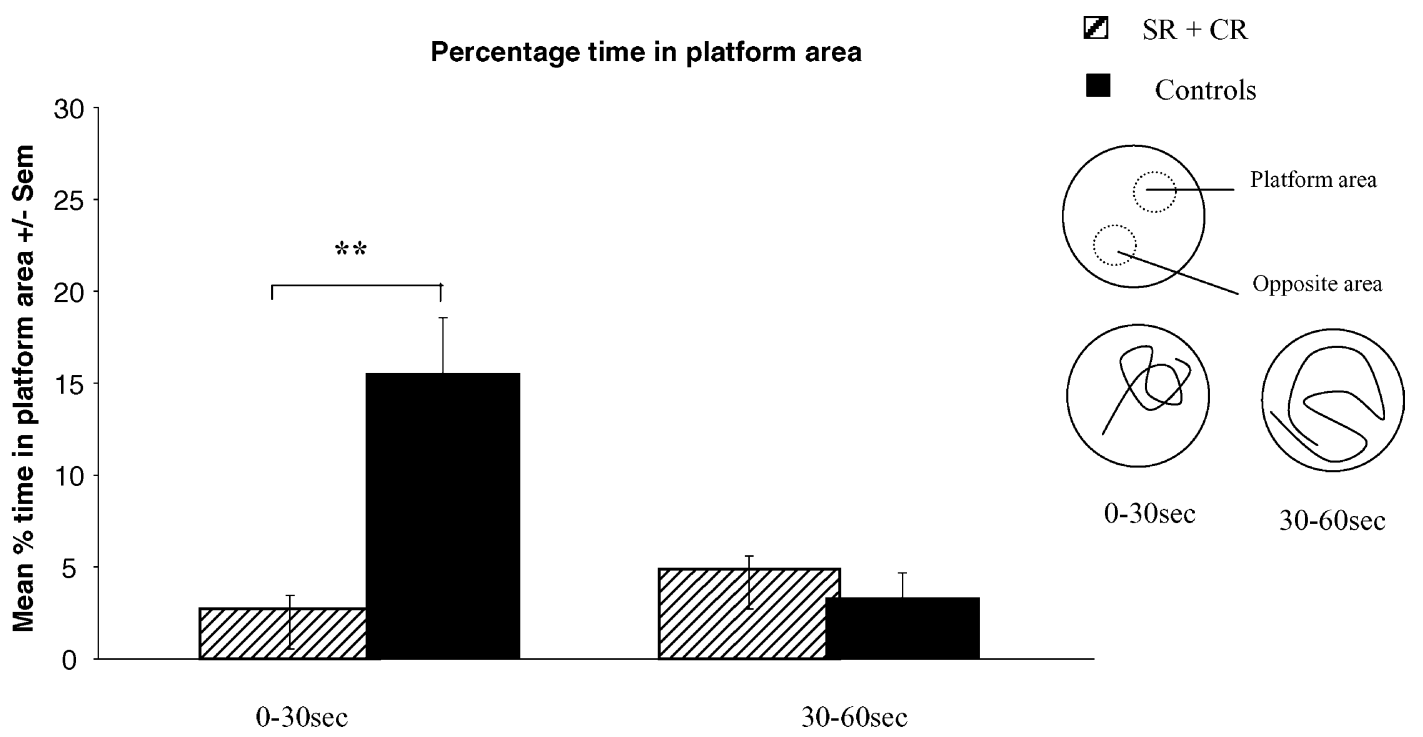

Percentage time in platform area in time bins

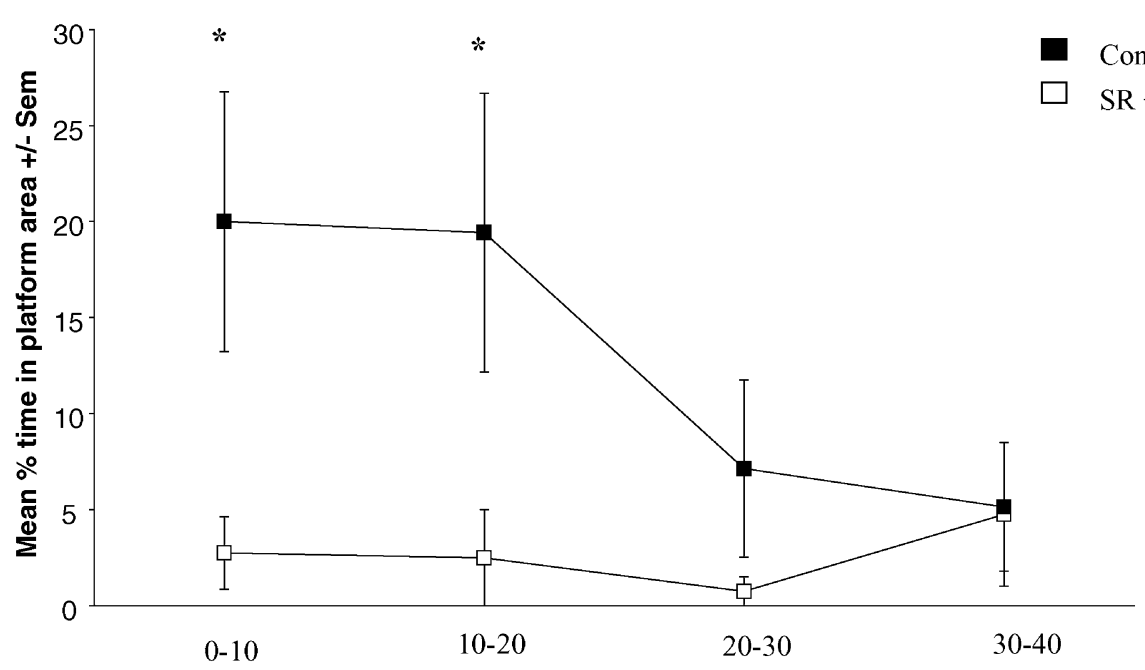

(b)

Time Bins (seconds)

Fig. 6. (a) Bar chart demonstrating the percentage time spent by control and start- and cue-rotated groups in the platform area (see inset) for the first and last $30 \mathrm{~s}$ of the retention phase. (b) A line chart displaying the percentage time spent by control and start- and cue-rotated groups in the platform area for the first $40 \mathrm{~s}$ of retention trial (in 10-s bins). Representative swim paths for the controls for the first and last $30 \mathrm{~s}$ are displayed in (a).

Retention of the water maze was assessed 7 days post-acquisition. Rats were randomly assigned to one of two groups: controls $(n=7)$ or start- and cue-rotated group $(n=8)$. Fig. 6a reveals that the control group spent significantly more time swimming in the platform area $(15.52 \pm 3 \%)$ than the start- and cue-rotated group $(2 \pm 1.45 \%)$ in the first $30 \mathrm{~s}(t=-4.191$, d.f. $=13$, $P<0.01)$. However, there was no significant difference $(t=0.417$, d.f. $=13, P>0.05)$ in the amount of time spent searching in the platform area by either the start- and cue-rotated or control group $(4.16 \pm 1.4 \%$ and $3.33 \pm 1.3 \%$, respectively) between 30 and $60 \mathrm{~s}$. We further analysed the first $40 \mathrm{~s}$ in $10-\mathrm{s}$ bins. We found that the controls spent significantly more time searching in the platform area in the first $10 \mathrm{~s}(t=-2.610$, d.f. $=13, P<0.05), 10-20 \mathrm{~s}$ $(t=-2.326$, d.f. $=13, P<0.05)$ when compared to the start- and cue-rotated group (see Fig. 6b). Rotation of the cues $180^{\circ}$ and changing the starting position impaired the search strategy of this group. Where did the start- and cue-rotated group search for the platform?

Fig. 7a demonstrates that the start- and cue-rotated group did not search significantly more in the opposite area when compared to controls in the first $30 \mathrm{~s}$ of retention $(t=$ -1.702 , d.f. $=13, P>0.05)$ or in the last $30 \mathrm{~s}(t=-0.304$, d.f. $=13, P>0.05)$. When the retention period was broken into 10-s bins (Fig. 7b) again no differences were observed between the groups. As before we analysed the percentage time spent by both groups in the 'panic corridor' to as- 
Percentage timein opposite area

(a)

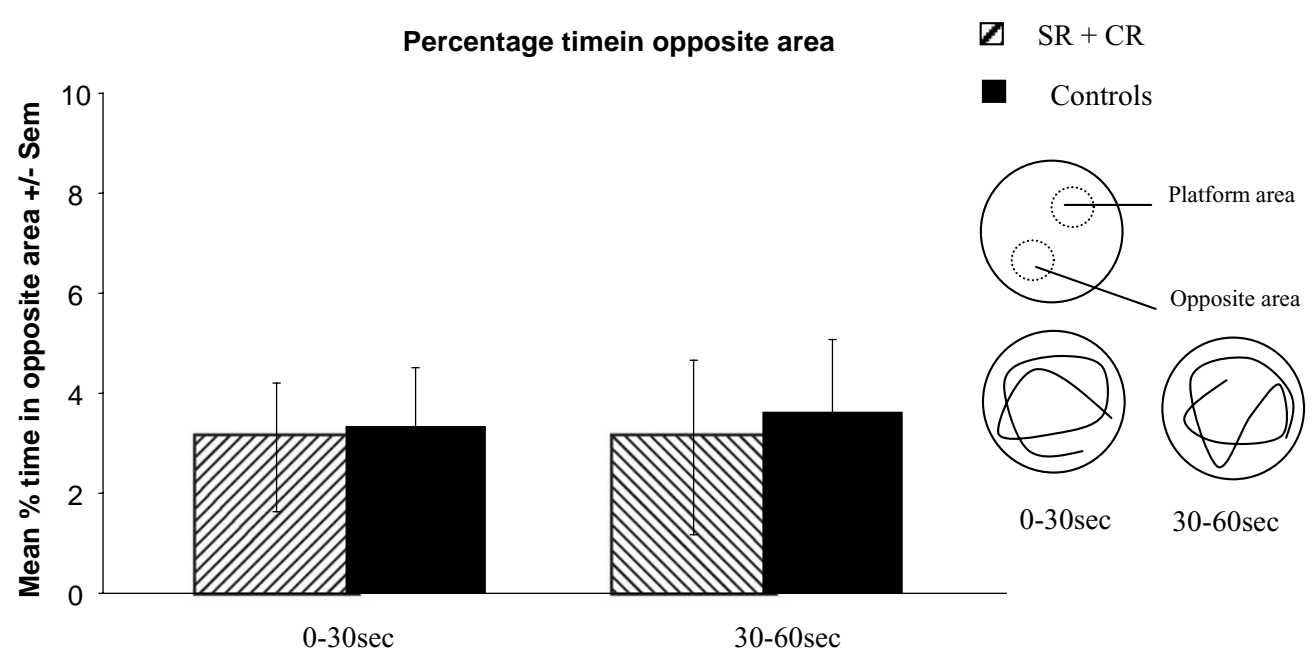

Percentage time in opposite area in timebins

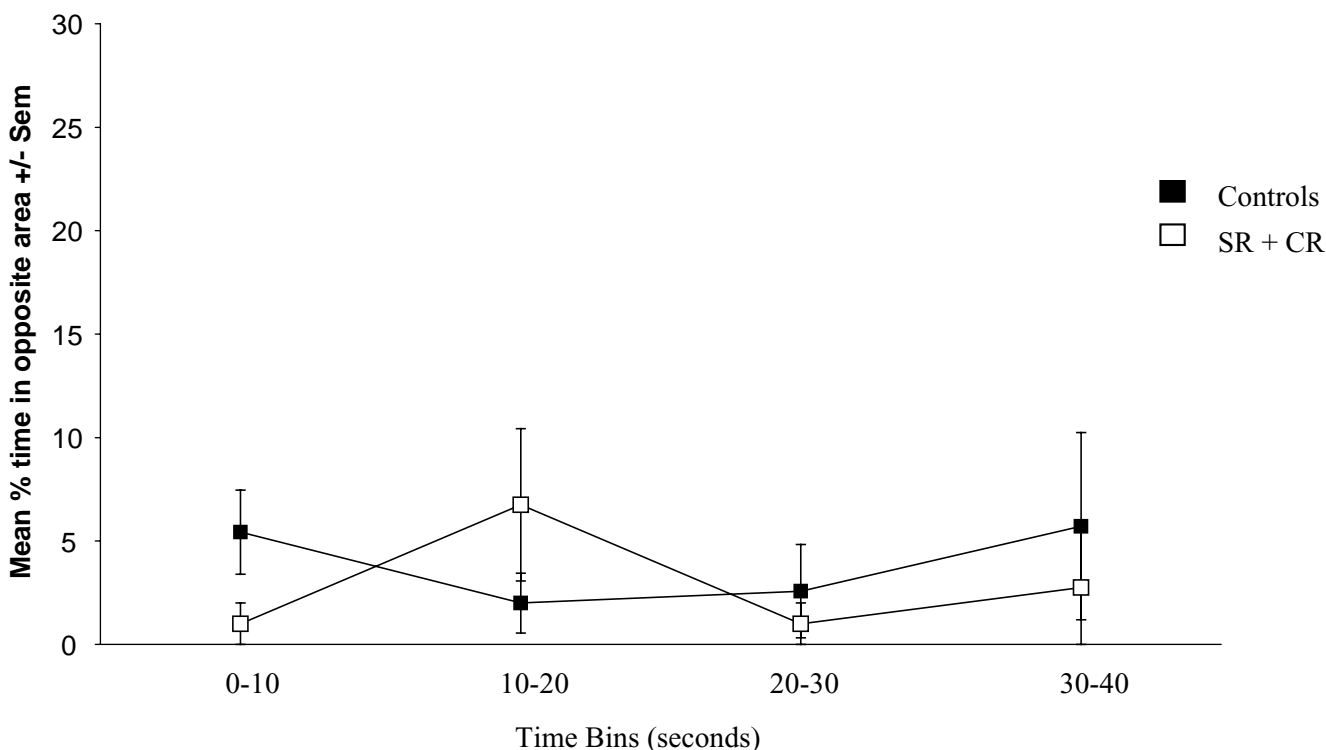

Fig. 7. (a) A bar chart displaying the percentage time spent by control and start- and cue-rotated groups in the opposite area (see inset) for the first and last $30 \mathrm{~s}$ of the retention phase. (b) A line graph demonstrating the percentage time spent in the opposite area by the control and start- and cue-rotated groups for the first $40 \mathrm{~s}$ of retention trial (in 10-s bins). Representative swim paths for the cue-rotated group for the first and last $30 \mathrm{~s}$ are displayed in (a).

sess confusion. Fig. 8a reveals that the start- and cue-rotated spent significantly more time $(77 \pm 9.1 \%)$ at the side of the pool than the control group $(22.57 \pm 6.1 \%)$ in the first $10 \mathrm{~s}(t=4.801$, d.f. $=13, P<0.01)$. However, unlike the start-rotated group (experiment 2) which remained at their starting position initially and then searched for the platform, the start- and cue-rotated group did not spend significantly more time at their starting position when compared to the control group $(t=-1.134$, d.f. $=13, P>0.05$, see Fig. 8b). Rather the start- and cue-rotated group swam around the side of the pool visiting all four areas equally in the first $10 \mathrm{~s}$ (see Fig. 8c). A one-way ANOVA confirmed that there was no overall significant difference in the mean percentage time spent by the start- and cue-rotated group in the four equivalent areas $(F=1.444$, d.f. $=3,31, P>$
0.05). A similar analysis was conducted for the controls (see Fig. 8d) revealing no differences.

\section{Discussion}

The experiments presented in this paper demonstrate that animals can easily retain knowledge of the water maze task 7 days post-acquisition. This confirms our recent findings using a training protocol similar to the one used in the current set of experiments [26]. Indeed previous research has indicated that knowledge of the task can be partially retained for at least 12 months particularly with 2 weeks of training [18].

In experiment 1 , we demonstrate that rotation of the distal cues leads to a rotation in the searching strategy of the 

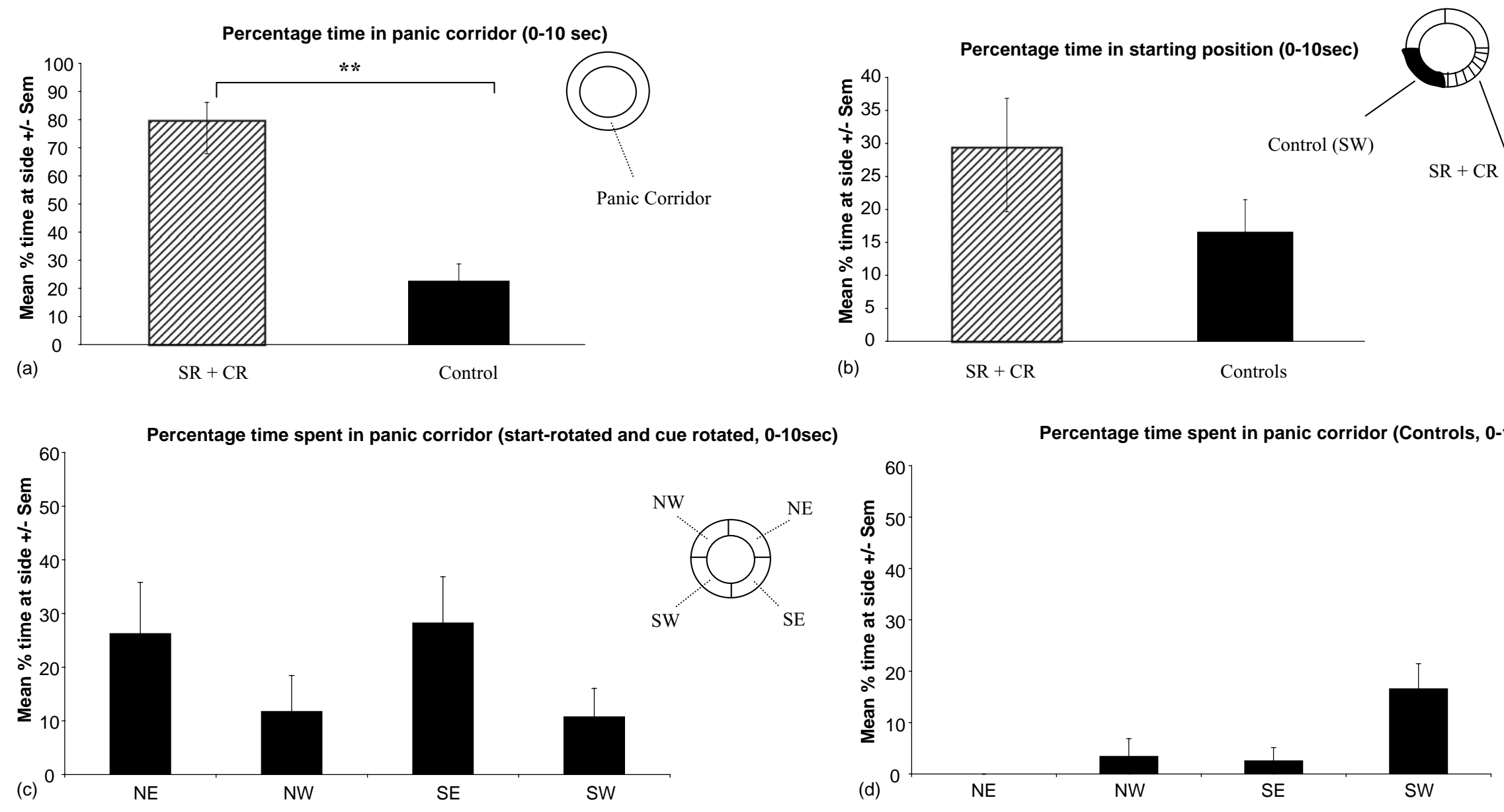

Fig. 8. (a and b) Bar charts displaying the percentage time spent swimming by the control and start- and cue-rotated groups in the panic corridor (a) and where they were placed into pool (b) for the first $10 \mathrm{~s}$. (c and d) Bar charts demonstrating the percentage time spent swimming in the four areas (see inset) of the panic corridor by the start- and cue-rotated group (c) and controls (d) for the first $10 \mathrm{~s}$. 
animals. This suggests that the association between the distal cues and the platform location built up during acquisition [19] is stored for at least 7 days. Retention of the water maze task is therefore dependent on the configuration of the distal cues and the location of the platform. However, the stored association between the distal cues and the platforms location (relevant to both the cue-rotated group and the control group) is time-dependent. We show that failure to locate the platform within $30 \mathrm{~s}$ leads to searching in other quadrants or along the side of the pool. We suggest that the standard use of a $60 \mathrm{~s}$ probe trial as a retention measure used by many authors (e.g. $[14,15,27])$ is too long and may miss subtle changes in search strategies. It has been well established that acquisition of the water maze task relies on distal cues [2] with research highlighting the importance of the hippocampus in this distal cue processing. Hippocampal-lesioned animals for example are impaired when they have to locate the platform based on both distal landmark cues $[28,29]$ and proximal cues [30]. However, Save and Poucet [28] argue that the processing of distal landmarks is mediated mainly by the hippocampus. Further evidence that suggests the involvement of the hippocampus in distal cue processing comes from single-cell recordings in the hippocampus. If distal cues are rotated, place fields of hippocampal neurons similarly rotate [31-33]. Recent evidence point to both the hippocampus and the entorhinal cortex in spatial-relational memory $[34,35]$. However, whether the hippocampus is directly involved in the retention of the associations between the platform location and distal cue configuration and for how long these associations are stored remain controversial (see [17]).

Rotation of the starting position also affected the searching strategy of the animals (experiment 2). We had predicted that animals trained from a fixed position would acquire an egocentric representation and would subsequently adopt a procedural strategy in the retention task when placed into the pool from the opposite starting point. However this did not seem to occur. The start-rotated group did not spent significantly more time searching in the opposite area when compared to the controls even in the initial 10-s retention period. Rather the start-rotated group remained at the pool edge (where they were placed) for at least $10 \mathrm{~s}$ and then commenced searching in the platform area. This suggests that following the initial $10 \mathrm{~s}$ of uncertainty animals then pursued a cue-based strategy. Further extensive training may lead to a shift in learning mechanisms from the cue to a response dominant strategy [36] which may in turn lead to a shift in what is retained by the animals. Alternatively complete absence of distal cues (see [21]) during the retention trial may lead animals to adopt a purely procedural strategy.

We further demonstrate that rotation of both the distal cues and also the starting position of the animals led to impairment in retention of the location of the platform (experiment 3). Again these findings were contrary to what we had predicted. We suggested that if a conflict arose between a procedural and a cue-based retention strategy, the cue-based strategy would be dominant. However, both rotations led to thigmotactic behaviour with animals swimming around the edge of the pool. A number of possibilities may account for the thigmotactic behaviour. First, animals may have simply forgotten the platform location with respect to the available stimuli, therefore, they revert to swimming along the side of the pool, similar to what is observed on the initial trial of the acquisition period. This increased swimming at the edge may be due to increased confusion or anxiety levels [22]. A second possibility is that the combination of starting rotation and cue rotation may give the perception of a novel environment. The usual variable used to explain the behavioural effects of novelty is fear or anxiety [24]. Devan et al. [24] observed an increased in thigmotaxis following caudate-putamen lesions and suggest that thigmotaxis may not be due to increased anxiety levels but rather a failure to initiate a competing response. That is, to move away from the wall and use the available cues to locate the platform. The increased thigmotaxis in our experiment may also be due to a failure to initiate a searching response.

The cue-based retention strategy did not dominate, as animals did not search in the opposite area to where the platform should have been. It has been suggested that solving navigational tasks using an allocentric map is flexible [2,21,37]. However, this experiment suggests that the allocentric map may not be as flexible as previously thought and retention of the distal cue and platform association is sensitive to disruption.

In conclusion, retention of the water maze task is based on the association between distal cues and the location of the platform. This association is built-up during acquisition and is the primary strategy used during retrieval. However, this association is fragile. The association is time-dependent; failure to successfully locate the platform based on this strategy within a period of time leads to alternative searching patterns. Although rotating the start position alone does not weaken this association it is sensitive to disruption by the combination of distal cue rotation and starting position rotation.

\section{Acknowledgements}

This work was supported by the Health Research Board of Ireland and the Department of Psychology, NUI Maynooth. We also thank Derek Walsh (Departmental Technician) for assistance.

\section{References}

[1] Abel T, Lattal KM. Molecular mechanisms of memory acquisition, consolidation and retrieval. Curr Opin Neurobiol 2001;11: $180-7$.

[2] Morris RGM. Spatial localisation does not require the presence of local cues. Learn Motiv 1981;12:239-60.

[3] Morris RGM. Development of a watermaze procedure for studying spatial learning in the rat. J Neurosci Methods 1984;11:47-60. 
[4] D'Hooge R, De Deyn PP. Applications of the Morris water maze in the study of learning and memory. Brain Res Rev 2001;36: 60-90.

[5] Davis S, Butcher SP, Morris RGM. The NMDA receptor antagonist D-2-amino-5-phosphonopentanoate (D-AP5) impairs spatial learning and LTP in vivo at intracerebral concentrations comparable to those that block LTP in vitro. J Neurosci 1992;12:21-34.

[6] Morris RGM, Garrud P, Rawlins JNP, O'Keefe J. Place navigation impaired in rats with hippocampal lesions. Nature 1982;297: 681-3.

[7] Sakimura K, Kutsuwada T, Ito I, Manabe C, Takayama E, Kushiya $\mathrm{E}$, et al. Reduced hippocampal LTP and spatial learning in mice lacking NMDA reduced e1 subunit. Nature 1995;373:151-5.

[8] Pearce JP, Roberts ADL, Good M. Hippocampal lesions disrupt navigation based on cognitive maps but not heading vectors. Nature 1998;396:75-7.

[9] Aggleton JP, Vann SD, Oswald CJP, Good M. Identifying cortical inputs to the rat hippocampus that subserves allocentric spatial processes: a simple problem with a complex answer. Hippocampus 2000;10:466-74.

[10] Gallistel CR. The organization of learning. Cambridge: MIT Press; 1990.

[11] Block F, Kenkel M, Schwarz M. Quinolinic acid lesions of the striatum induces impairment in spatial learning and motor performance in rats. Neurosci Lett 1993;149:126-8.

[12] Nadel L, Samsonovich A, Ryan L, Moscovitch M. Multiple trace theory of human memory: computational, neuroimaging and neuropsychological results. Hippocampus 2000;10:352-68.

[13] O'Keefe J, Nadel L. The hippocampus as a cognitive map. Oxford: Oxford University Press; 1978.

[14] Riedal G, Micheau J, Lam AGM, Roloff EVL, Martin SJ, Bridge H, et al. Reversible neural inactivation reveals hippocampal participation in several memory processes. Nat Neurosci 1999;2:898-905.

[15] De Quervain DJF, Roozendaal B, McGaugh JL. Stress and glucocorticoids impair retrieval of long-term spatial memory. Nature 1998;394:787-90.

[16] Bolhuis JJ, Stewart CA, Forrest EM. Retrograde amnesia and memory reactivation in rats with ibotenate lesions to the hippocampus or subiculum. Q J Exp Psychol B 1994;47:129-50.

[17] Mumby DG, Astur RS, Weisend MP, Sutherland RJ. Retrograde amnesia and selective damage to the hippocampal formation: memory for places and object discrimination. Behav Brain Res 1999;106:97107.

[18] Van Groen T, Kadish I, Wyss MJ. Old rats remember old tricks memories of the water maze persist for 12 months. Behav Brain Res 2002;136:247-55.

[19] Cimadevilla JM, Wesierska M, Fenton AA, Bures J. Inactivating one hippocampus impairs avoidance of a stable room-defined place during dissociation of arena cues from room cues by rotation of the arena. Proc Natl Acad Sci USA 2001;98:3531-6.
[20] Whishaw IQ. Place learning in hippocampal rats and the path integration hypothesis. Neurosci Biobehav Rev 1998;22(2):209-20.

[21] Moghaddam M, Bures J. Contribution of egocentric spatial memory to place navigation of rats in the Morris water maze. Behav Brain Res 1996;78:121-9.

[22] Johansson I-M, Birzniece, Lindblad C, Olsson T, Backstrom T Allopregnanolone inhibits learning in the water maze. Brain Res. 2002;934:125-31.

[23] Barnett SA. The rat: A study in Behaviour. Chicago: Aldine, 1963: 288

[24] Devan BD, McDonald RJ, White NM. Effects of medial and lateral caudate-putamen lesions on place- and cue-guided behaviors in the water maze: relation to thigmotaxis. Behav Brain Res 1999;100:5-14.

[25] Devan BD, Goad EH, Petri HL. Dissociation of hippocampal and striatal contributions to spatial navigation in the warer maze. Neurobiol Learn Mem 1996;66:305-23.

[26] Commins S, Cunningham L, Harvey D, Walsh D. Massed but not spaced training impairs spatial memory. Behav Brain Res 2003;139:215-23.

[27] Roozendaal B, Griffith QK, Buranday J, De Quervain DJ, McGaugh JL. The hippocampus mediates glucocorticoid-induced impairment of spatial memory retrieval: dependence on the basolateral amygdala Proc Natl Acad Sci USA 2003;100(3):1328-33.

[28] Save E, Poucet B. Involvement of the hippocampus and associative parietal cortex in the use of proximal and distal landmarks for navigation. Behav Brain Res 2000;109(2):195-206.

[29] Jarrard LE. On the role of the hippocampus in learning and memory in the rat. Behav Neural Biol 1993;60(1):9-26.

[30] Sakamoto T, Okaichi H. Effects of fimbria-fornix lesions on rats' use of intramaze stimuli in the water maze. Psychobiology 1998;26: 231-9.

[31] Cressant A, Muller RU, Poucet B. Remapping of place cell firing patterns after maze rotations. Exp Brain Res 2002;143(4):470-9.

[32] Kubie JL, Ranck Jr JB. Sensory-behavioral correlates in individual hippocampus neurons in three situations: space and context. In: Seifert W, editor. Neurobiology of the hippocampus. New York: Academic Press; 1983. p. 433-47.

[33] O'Keefe J, Speakman A. Single unit activity in the rat hippocampus during a spatial memory task. Exp Brain Res 1987;68:1-27.

[34] Ramos JMJ. Retention of spatial information in hippocampally damaged rats overtrained on the cartographic task. Brain Res 2000;879:200-3.

[35] Kaut KP, Bunsey MD. The effects of lesions to the rat hippocampus or rhinal cortex on olfactory and spatial memory: retrograde and anterograde findings. Cog Affect Behav Neurosci 2001;1(3):270-86.

[36] Packard MG, McGaugh JL. Inactivation of hippocampus or caudate nucleus with lidocaine differentially affects expression of place and response learning. Neurobiol Learn Mem 1996;65:65-72.

[37] Panakhova E, Buresova O, Bures J. Persistence of spatial memory in the Morris water tank task. Int J Psychophysiol 1984;2:5-10. 